\title{
Bidirectional Relationship between Opioids and Disrupted Sleep: Putative Mechanisms
}

\author{
DD. Eacret, S.C. Veasey, and (DJ.A. Blendy \\ Departments of Systems Pharmacology and Translational Therapeutics (D.E., J.A.B.) and Medicine (S.C.V.), Perelman School of \\ Medicine, University of Pennsylvania, Philadelphia, Pennsylvania
}

Received December 6, 2019; accepted March 12, 2020

\section{ABSTRACT}

Millions of Americans suffer from opiate use disorder, and over 100 die every day from opioid overdoses. Opioid use often progresses into a vicious cycle of abuse and withdrawal, resulting in very high rates of relapse. Although the physical and psychologic symptoms of opiate withdrawal are welldocumented, sleep disturbances caused by chronic opioid exposure and withdrawal are less well-understood. These substances can significantly disrupt sleep acutely and in the long term. Yet poor sleep may influence opiate use, suggesting a bidirectional feed-forward interaction between poor sleep and opioid use. The neurobiology of how opioids affect sleep and how disrupted sleep affects opioid use is not well-understood. Here, we will summarize what is known about the effects of opioids on electroencephalographic sleep in humans and in animal models. We then discuss the neurobiology interface between reward-related brain regions that mediate arousal and wakefulness as well as the effect of opioids in sleep-related brain regions and neurotransmitter systems. Finally, we summarize what is known of the mechanisms underlying opioid exposure and sleep. A critical review of such studies, as well as recommendations of studies that evaluate the impact of manipulating sleep during withdrawal, will further our understanding of the cyclical feedback between sleep and opioid use.

\section{SIGNIFICANCE STATEMENT}

We review recent studies on the mechanisms linking opioids and sleep. Opioids affect sleep, and sleep affects opioid use; however, the biology underlying this relationship is not understood. This review compiles recent studies in this area that fill this gap in knowledge.

\section{Introduction}

Every day in the United States, there are over 130 deaths due to opioid overdose (Hedegaard et al., 2020). The associated healthcare, lost productivity, and criminal justice involvement costs approximately $\$ 80$ billion per year (Scholl et al., 2018). Opioids are so addictive that $10 \%-30 \%$ of patients that are prescribed opioids misuse them and/or develop an opioid use disorder (Vowles et al., 2015). Intense withdrawal symptoms can prevent successful long-term abstinence from opioids. One such contributing opiate withdrawal symptom is disrupted sleep (Beswick et al., 2003). Poor sleep is a symptom of both opioid use (Hartwell et al., 2014) and withdrawal (Sharkey et al., 2009) and is a reason for relapse back to opioid use

This work was supported by grants from the National Institute of Health National Institute on Drug Abuse [Grants R01DA41180 and T32DA028874.] (J.A.B. and D.E. respectively) and National Heart, Lung, and Blood institute [Grant R01HL12331] (S.C.V.).

https://doi.org/10.1124/mol.119.119107.
(Maulik et al., 2002; Lydon-Staley et al., 2017). Specifically, more than $75 \%$ of patients on prescription opioids or methadone maintenance therapy were categorized as poor sleepers (Stein et al., 2004; Hartwell et al., 2014). Disrupted sleep leads to increases in negative affect (Sagaspe et al., 2006) and poorer cognitive abilities (Durmer and Dinges, 2005; Lim and Dinges, 2008), both of which have been associated with increased tendency to relapse. In addition, sleep disruption can lead to decreased pain tolerance (Roehrs et al., 2006), and if this occurs during opioid withdrawal, it could also contribute to high relapse rates. There may be a common vicious cycle of opioid use that induces sleep disturbances, further increasing usage of opioids. For example, poor sleep can be a risk factor for drug use, especially in adolescents. In a study of 60,000 adolescents, poorer sleep was associated with a higher odds ratio for risk of drug use (Weaver et al., 2018), and adolescents with circadian abnormalities (with secondary sleep disturbances) have increased vulnerability for addiction (Logan

ABBREVIATIONS: A2AR, adenosine 2A receptor; CTOP, D-Phe-Cys-Tyr-D-Trp-Orn-Thr-Pen-Thr-NH2; D1R, dopamine D1 receptor; D2R, dopamine D2 receptor; DREADD, designer receptor exclusively activated by designer drugs; EEG, electroencephalography; KOR, $\kappa$-opioid receptor; MOR, $\mu$-opioid receptor; MPRF, medial pontine reticular formation; NAc, nucleus accumbens; NREM, non-REM; PDYN, prodynorphin; PVT, paraventricular nucleus of the thalamus; REM, rapid eye movement; RMTg, rostromedial tegmental nucleus; SWS, slow-wave sleep; TH, tyrosine hydroxylase; VLPO, ventrolateral preoptic area; VTA, ventral tegmental area. 
et al., 2018). Understanding the biology linking sleep and opioids as well as targeting therapeutics to improve sleep during opioid withdrawal are necessary to help reduce the burden of opioids on society.

Currently there are few studies examining opioid use and sleep, and any mechanistic studies that do exist focus on the acute effects of opioids on sleep and wakefulness. However, recent technologies, such as optogenetics, chemogenetics, and fiber photometry, can be combined with electroencephalography (EEG) to give a more detailed picture of specific cell types within the brain that modulate sleep and wakefulness (summarized in Table 1). Many of these studies show that brain regions and neurotransmitter systems associated with reward greatly contribute to arousal and may mechanistically contribute to the sleep/wake state of an animal. These studies thus provide potential targets for opioid regulation of sleep states.

\section{Effects of Opioids on Human Sleep}

One of the earliest descriptions of opioid effects on sleep in humans involved four researchers who examined the effects of heroin on their own sleep. Heroin rapidly converts to morphine upon transit across the blood-brain barrier. After three nights of baseline sleep, EEG recordings were made after subcutaneous injection of heroin before sleep for three or seven consecutive nights (Lewis et al., 1970). Acute administration of heroin resulted in suppression of rapid-eye-movement (REM) sleep by $50 \%$ with smaller reductions after consecutive nights of heroin administration. Upon withdrawal, REM sleep rebound was present and persisted for days. Additionally, heroin administration suppressed deep non-REM (NREM) sleep [also known as slow-wave sleep (SWS)] while increasing both lighter NREM sleep and transitions to wakefulness and drowsiness (Lewis et al., 1970). A similar acute effect of suppressing deep NREM sleep and increasing light sleep has been seen for both long-acting morphine and methadone (Dimsdale et al., 2007). Effects appear quite similar for acute usage of short-acting morphine. Specifically, intravenous administration of morphine sulfate to healthy young adult

TABLE 1

Manipulation of opioid/reward-related neurons and their effects on sleep A table of recent studies that manipulate neurons in reward-related brain regions and monitor sleep stages with electroencephalography. Optogenetic and chemogenetic manipulations allow exploration in a cell type-specific manner of whether certain neurons are wake-promoting or sleep-promoting.

\begin{tabular}{|c|c|c|c|c|c|}
\hline Brain Region & Cell Type & Technique & Activate + or Inhibit - & Sleep Effect & Author \\
\hline \multirow[t]{8}{*}{ NAc } & \multirow[t]{3}{*}{ D1R } & Optogenetics & + & $\uparrow$ wake & \multirow[t]{5}{*}{ Luo et al., 2018} \\
\hline & & DREADDs & + & $\uparrow$ wake & \\
\hline & & DREADDs & - & $\uparrow \mathrm{NREM}$ & \\
\hline & \multirow[t]{2}{*}{ D2R } & DREADDs & + & $\uparrow \mathrm{NREM}$ & \\
\hline & & DREADDs & - & $\uparrow$ wake & \\
\hline & \multirow[t]{3}{*}{$\mathrm{A} 2 \mathrm{AR}$} & DREADDs & + & $\uparrow$ SWS & \multirow[t]{3}{*}{ Oishi et al., 2017} \\
\hline & & Optogenetics & + & $\uparrow \mathrm{SWS}$ & \\
\hline & & DREADDs & - & $\downarrow$ SWS & \\
\hline \multirow[t]{7}{*}{ VTA } & \multirow[t]{3}{*}{$\mathrm{TH}$} & DREADDs & - & $\begin{array}{c}\downarrow \text { Wake } \\
\uparrow \mathrm{NREM} \\
\uparrow \mathrm{REM}\end{array}$ & \multirow[t]{2}{*}{ Eban- Rothschild et al., 2016} \\
\hline & & Optogenetics & + & $\uparrow$ wake & \\
\hline & & DREADDs & - & $\begin{array}{l}\downarrow \text { wake } \\
\uparrow \text { NREM }\end{array}$ & Yang et al., 2018 \\
\hline & Glutamate (Vglut2) & DREADDs & + & $\uparrow$ wake & Yu et al., 2019 \\
\hline & GABA (Vgat) & DREADDs & + & $\uparrow$ NREM & \\
\hline & \multirow[t]{2}{*}{ GABA (Gad67) } & DREADDs & + & $\uparrow$ NREM & \multirow[t]{2}{*}{ Chowdhury et al., 2019} \\
\hline & & Optogenetics & - & $\uparrow$ wake & \\
\hline $\mathrm{VTA} \rightarrow \mathrm{LH}$ & GABA (Gad67) & Optogenetics & + & $\uparrow \mathrm{NREM}$ & \\
\hline \multirow[t]{2}{*}{$\mathrm{VTA} \rightarrow \mathrm{LH}$} & \multirow[t]{2}{*}{ GABA (Vgat) } & DREADDs & - & $\uparrow$ wake & \multirow{2}{*}{ Yu et al., 2019} \\
\hline & & Optogenetics & + & $\uparrow$ NREM & \\
\hline \multirow[t]{3}{*}{ PVT } & \multirow[t]{3}{*}{ Glutamate (CAMKIIa) } & DREADDs & - & $\downarrow$ wake & \multirow[t]{3}{*}{ Ren et al., 2018} \\
\hline & & Lesion & - & $\downarrow$ wake & \\
\hline & & Optogenetics & + & $\uparrow$ wake & \\
\hline \multirow[t]{3}{*}{ RMTg } & \multirow[t]{3}{*}{ Nonspecific, mostly GABA } & DREADDs & + & $\uparrow \mathrm{NREM}$ & \multirow[t]{3}{*}{ Yang et al., 2018} \\
\hline & & DREADDs & - & $\begin{array}{l}\downarrow \text { NREM } \\
\uparrow \text { wake }\end{array}$ & \\
\hline & & Lesion & - & $\begin{array}{l}\downarrow \text { NREM } \\
\downarrow \text { REM }\end{array}$ & \\
\hline \multirow[t]{5}{*}{ Dorsal raphe } & \multirow[t]{2}{*}{$\mathrm{TH}$} & Optogenetics & + & $\uparrow$ wake & \multirow[t]{2}{*}{ Cho. et al., 2017} \\
\hline & & DREADDs & - & $\downarrow$ wake & \\
\hline & \multirow[t]{3}{*}{ SERT } & Optogenetics & + (burst) & $\begin{array}{l}\downarrow \text { NREM } \\
\downarrow \text { REM }\end{array}$ & \multirow[t]{3}{*}{ Oikonomou et al., 2019} \\
\hline & & Optogenetics & $+($ tonic $)$ & $\uparrow$ NREM & \\
\hline & & Lesion & - & $\begin{array}{c}\uparrow \text { wake } \\
\downarrow \text { NREM } \\
\downarrow \text { REM }\end{array}$ & \\
\hline POA & PDYN & Optogenetics & + & $\begin{array}{l}\downarrow \text { wake } \\
\uparrow \text { NREM }\end{array}$ & Chung et al., 2017 \\
\hline
\end{tabular}

CAMKIIa, Calcium/calmodulin-dependent protein kinase type II subunit alpha; Gad67, Glutamate decarboxylase; LH, lateral hypothalamus; SERT, serotonin transporter. POA, preoptic area; Vgat, vesicular GABA transporter; Vglut2, vesicular glutamate transporter 2. 
subjects significantly increased light NREM sleep and suppresses deep NREM sleep (67\% reduction) and REM sleep (25\% reduction) (Shaw et al., 2005). Chronic methadone users showed light (poor quality) sleep and, in addition, shorter sleep times and greater fragmentation of sleep (Xiao et al., 2010). A constant and overnight infusion of remifentanil decreased REM in healthy human volunteers (Bonafide et al., 2008). Patients with chronic noncancer pain with the single nucleotide polymorphism in the $\mu$-opioid receptor (MOR) gene OPRM1 118-GG reported increased sleep disruption and poorer sleep patterns after 3 months of opioid treatment compared with patients with the standard 118-AA genotype (Margarit et al., 2019). Thus, poor sleep is commonly observed with opioid use, and genetics may contribute to the extent of the problem.

Despite the observations that opioids profoundly affect sleep quality, withdrawal from opioid use also has significant effects on sleep architecture. In an early study of heroin users, acute withdrawal resulted in greater sleep/wake transitions and less REM sleep (Howe et al., 1981). In individuals on a methadone maintenance program, acute withdrawal resulted in increased deep NREM sleep and REM sleep for at least 10 weeks (Martin et al., 1973). Whether sleep ever normalizes remains unknown. These experiments show that opioids disrupt sleep in humans, and sleep does not normalize with available medication-assisted therapy for opioid use disorders. Thus, a better understanding of the mechanisms involved in opioid dysregulation of sleep is critical to improve treatments for this aspect of withdrawal.

\section{Preclinical Sleep Studies on the Effects of Opioids}

Acute administration of morphine in cats resulted in significant suppression of both NREM and REM sleep with resultant increased wakefulness, particularly in the first few hours after administration (De Andrés and Caballero, 1989). This increased wake (albeit less pronounced) persisted across 2 weeks of daily administration (De Andrés and Caballero, 1989). Interestingly, upon withdrawal after 2 weeks of morphine, the cats, like humans, showed increased NREM and REM sleep (De Andrés and Caballero, 1989). Similarly, morphine administration in rats profoundly suppresses REM sleep acutely and chronically, and withdrawal results in a sustained increase in REM sleep (Khazan and Colasanti, 1972).

Animal studies have brought some insight into the mechanisms of opioid effects on sleep. The medial pontine reticular formation (MPRF) in the brainstem is a region involved in both nociception and REM sleep, and intriguingly, morphine microinjected into the MPRF in the cat suppressed REM sleep (Keifer et al., 1992), which is mediated by activation of MORs in the MPRF (Cronin et al., 1995). Furthermore, direct injection of morphine in the MPRF actually suppressed release of the pro-REM sleep neurotransmitter acetylcholine from the lateral dorsal tegmentum (Lydic et al., 1993). The effects of opioids on sleep are far more complicated and involve more than just the MPRF brainstem effect of morphine suppressing REM sleep because there are additional effects of opioids in several wake-active brain regions as well as sleep-active regions.
Mechanistically, one way acute morphine inhibits slow-wave sleep is via $\mu$-opioid receptors located on GABAergic neurons in the ventrolateral preoptic area (VLPO), a well-known sleep-promoting brain region (Wang et al., 2013). Rats given a subcutaneous injection of morphine showed increased wakefulness and decreased NREM and REM for a 3-hour period after injection (Wang et al., 2013). In brain slices ex vivo, morphine hyperpolarized the membrane potential in the VLPO and inhibited firing of these sleep-promoting neurons. This effect was dependent on activation of MORs because the MOR antagonist CTOP prevents morphineinduced hyperpolarization (Wang et al., 2013). However, morphine still partially inhibited the firing of VLPO sleeppromoting neurons even in the presence of CTOP. Full activity was restored in the presence of both morphine and CTOP as well as the $\kappa$-opioid receptor (KOR) antagonist, norbinaltorphimine, indicating morphine-induced wakefulness is mediated by both $\mu$ - and $\kappa$-opioid receptors in the VLPO (Wang et al., 2013). In vivo, morphine-induced wakefulness from a 1-mg/kg subcutaneous injection was blocked by CTOP injection into the VLPO, whereas CTOP injection in a vehicle-treated animal did not affect sleep/wakefulness (Wang et al., 2013). $\mu$-Opioid receptor mRNA is expressed in the majority of sleep-promoting neurons in the VLPO, and MOR agonists infused into the VLPO increase wakefulness (Greco et al., 2008). Translational profiling from preoptic neurons followed by RNA sequencing showed that the prodynorphin (PDYN) gene is highly expressed in GABAergic preoptic area neurons (Chung et al., 2017). Prodynorphin encodes for the opioid peptide dynorphin, and optogenetic activation of these PDYN neurons decreases wakefulness and increases NREM (Chung et al., 2017). Local morphine, however, can have sleep/wake effects beyond the VLPO. Injecting morphine into the rostromedial tegmental nucleus (RMTg), also known as the tail of the ventral tegmental area (VTA), hyperpolarizes and inactivates these neurons and decreases NREM and REM while increasing wake in a 3-hour period (Yang et al., 2018).

There is a dearth of research that evaluates the impact of chronic opioids or withdrawal on sleep. One of the only studies to do so found that rat self-administration of heroin over a 6-hour period each day reversed the sleep-wake cycle across 14 days of acquisition. Here, rats were placed in the selfadministration chambers from 11:00 to 17:00 and in their home cage for the other 18 hours, and EEG/electromyography was recorded continuously. This paradigm resulted in both "low drug takers" and "high drug takers," but regardless of the amount of drug self-administered, heroin increased time awake during the light cycle and decreased time awake during the dark cycle compared with saline-infused rats (Coffey et al., 2016). During abstinence, wake and NREM returned to baseline circadian rhythms, whereas REM sleep maintained its abnormalities for 3-6 days (Coffey et al., 2016). These studies show a general effect of opioids to reduce or disrupt sleep; however, more studies in this area are needed, especially mechanistic studies assessing the impact of chronic opioid use and extended withdrawal on sleep. 


\section{Preclinical Studies on How Sleep Affects Opioid Response}

In addition to the effects of opioids on sleep, as described above, findings from several studies demonstrate that disruption of sleep can affect pain sensitivity and response to opioids, leading to a bidirectional feedback loop between sleep and opioids. For example, poor sleep causes many negative symptoms that likely contribute to high rates of opioid relapse, including increased pain sensitivity (Roehrs and Roth, 2005). In humans, sleep deprivation for two consecutive nights lowers thresholds for mechanical pain, and effects are greater for total sleep loss relative to effects of depriving specifically NREM or REM sleep (Onen et al., 2001). Similarly, acute short-term sleep loss (9 hours) in mice resulted in shorter hot-plate withdrawal latencies, which normalize after recovery sleep (Alexandre et al., 2017). Moreover, REM sleep deprivation in the rat across 1 or 2 days lowered the thresholds for mechanical and chemical pain and, importantly, reduced the antinociceptive effect of morphine injected into the periacquaductal gray (Tomim et al., 2016). A pattern of sleep commonly observed in modern societies is chronic short sleep. Chronic short sleep in mice also results in lower pain thresholds in a durationdependent fashion (Alexandre et al., 2017). Interestingly, heightened pain sensitivity is a consequence of reduced arousal after sleep loss. Specifically, pain thresholds could be normalized with wake-promoting drugs (e.g., caffeine and modafinil), whereas morphine had less effect in countering pain after sleep loss. Together, these findings indicate that the sleep/wake state of an animal affects analgesic properties of opioids (Alexandre et al., 2017). In support of an arousal and pain threshold connection, the hyperalgesia after 96 hours of REM sleep deprivation resulted in loss of tyrosine hydroxylase (TH), the rate-limiting enzyme for dopamine synthesis, and pain threshold could be normalized by administering dopamine systemically prior to testing (Skinner et al., 2011). In contrast, the hyperalgesia after REM sleep deprivation in the rat did not influence regional MOR receptor binding throughout the brain (Nascimento et al., 2007). Poor sleep heightens pain sensitivity (Roehrs et al., 2006), resulting in the requirement for more pain medications. Therefore, improving sleep may be beneficial in reducing tolerance to pain medication, thereby contributing to reductions in opioid use and abuse.

Most studies examining the effect of sleep on opioid use and response to opioids are indirect. Animal studies show that lack of sleep can affect brain activity in regions that modulate opioid response, such as the medial habenula. Sleep fragmentation for 5 days increases the activity of habenula neurons (Ge et al., 2019), and habenula activity is linked to negative affect during morphine withdrawal (Valentinova et al., 2019). In addition, an alpha-3 beta- 4 nicotinic receptor nicotinic receptor antagonist injected into the medial habenula attenuated self-administration of morphine in rats (Glick et al., 2006). Nicotinic receptors in the habenula may indirectly modulate the effects of sleep fragmentation on the extent of negative effects associated with morphine exposure.

The opioid system may also have a role in sleep disruption beyond that resulting from drug use. Disruptions in sleep due to stress are mediated by the KOR, as a $30 \mathrm{mg} / \mathrm{kg}$ injection of the KOR antagonist JDTic improved recovery sleep after stress and reversed the expression of the circadian clock gene mPer2 in the nucleus accumbens (Wells et al., 2017).

\section{Likely Mechanisms of Opioid Effects on Sleep and Areas of Future Studies}

Several neurotransmitters and neuromodulators have been shown to be present on wake-activated or sleep-activated neurons, whose activity and signaling mechanisms can also be affected by opioids. Here we summarize recent studies to best determine which neurotransmitters and neuromodulators in various brain regions most likely underlie the effects of opioids on sleep/wake. Schematics of these systems are shown in Figures 1 and 2.

Dopamine. The mesolimbic dopamine pathway plays a key role in mediating rewarding properties of drugs of abuse. Patients with disorders that decrease dopamine levels and function have sleep disturbances (Louter et al., 2012). Chronic morphine enhances the activity of dopamine neurons in the VTA via inhibitory $\mu$-opioid receptors on GABAergic interneurons (Nestler, 2004). VTA soma size decreased, and neuronal excitability increased after two 25-mg subcutaneous morphine pellets that were implanted for 4 days in mice (Mazei-Robison et al., 2011). Decreased volume in the VTA was also observed in postmortem brain slices from human heroin overdose subjects (Mazei-Robison et al., 2011). Dopamine neurons (marked by expression of tyrosine hydroxylase; $\mathrm{TH}+$ ) in the VTA are more active during wake and REM sleep than NREM sleep (Eban-Rothschild et al., 2016). Optogenetically activating TH+-VTA neurons induces and maintains wakefulness, whereas chemogenetically inhibiting these neurons increases sleep and decreases wakefulness (EbanRothschild et al., 2016). In a similar study, designer receptor exclusively activated by designer drugs (DREADD) inhibition of VTA dopamine neurons decreased wakefulness and increased NREM without affecting REM sleep (Yang et al., 2018). In addition, inhibiting dopamine neurons in the substantia nigra decreased wakefulness and increased NREM (Yang et al., 2018). It is of interest that activating dopamine neurons in the VTA causes emergence from anesthesia (Taylor et al., 2016).

$\mathrm{TH}+$ neurons in the VTA may be a locus for the intersection of sleep and reward. The VTA projects to the nucleus accumbens (NAc) via the mesolimbic dopamine pathway. Optogenetic activation of dopamine D1 receptor (D1R)expressing neurons in the NAc quickly produces wakefulness and extends arousal (Luo et al., 2018). NAc D1R neurons mainly target the substantia nigra, sparsely target the VTA, and can locally inhibit D2R-containing neurons (Luo et al., 2018). Chemogenetic inhibition of nucleus accumbens D2R neurons increased wakefulness and decreased NREM and REM 2 hours after injection of the chemical activator clozapine $N$-oxide injection. Activation of D2R neurons is sleeppromoting, as chemogenetic activation of these receptors increased NREM and decreased wakefulness (Luo et al., 2018). Thus, nucleus accumbens D1R and D2R neurons have opposing effects on sleep/wakefulness. A large subset of dorsal raphe/midbrain neurons are also dopaminergic and project to the VTA (Mcdevitt et al., 2014), and these neurons express $\mu$-opioid receptors ( $\mathrm{Li}$ et al., 2016). Optogenetically activating dorsal raphe dopamine neurons is arousing in mice, whereas chemogenetically silencing these neurons induces sleep (Cho et al., 2017). Activity of dopaminergic dorsal raphe neurons from calcium imaging paralleled EEG sleep states in that there was increased activity during wake and decreased 

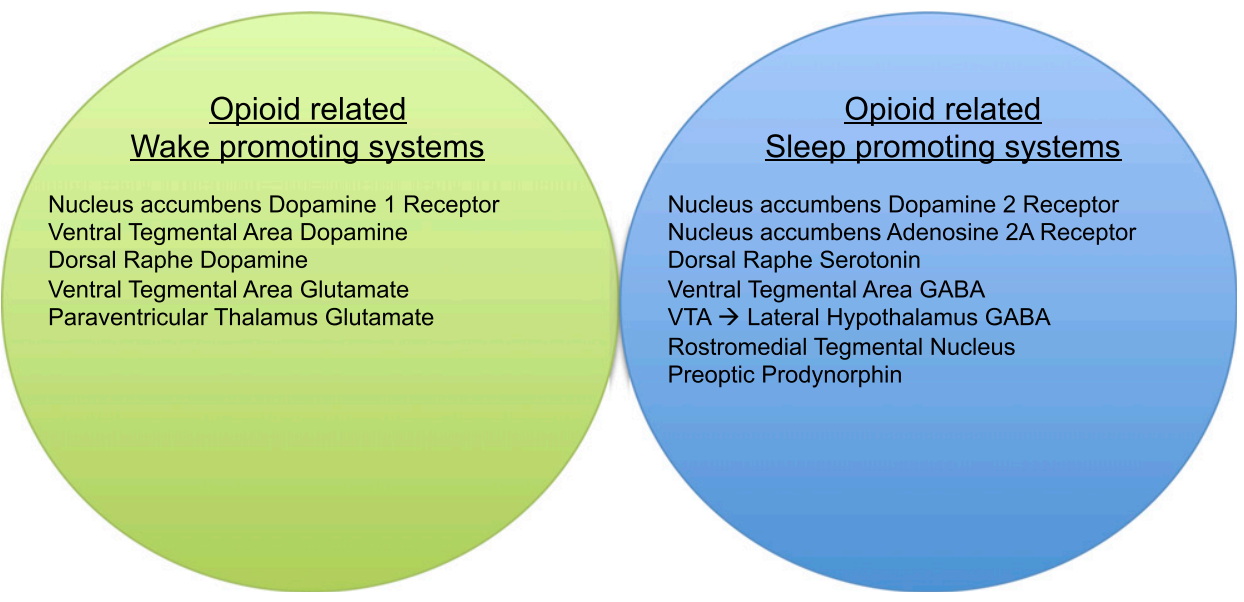

Fig. 1. Opioid-related wake-promoting vs. opioid-related sleep-promoting systems. These regions and cell types have been shown in this review to be related to opioids and affect sleep. The wake-promoting systems are generally activated by opioids, and the sleep-promoting systems are generally inhibited by opioids.

activity during sleep (Cho et al., 2017). These neurons are also activated in response to rewarding stimuli, such as consuming chocolate (Cho et al., 2017). Overall, it appears dopamine neurons across multiple different brain regions contribute to arousal of an animal, and at least components of this dopamine system are engaged by opioid use. Current studies have only examined dopamine's arousal properties immediately after manipulation, and future studies focused on chronic or long-term activation are necessary to determine their role in opioid modulation of sleep/wake states.

Orexin/Hypocretin. Orexin/hypocretin neurons were discovered in the late 1990s (de Lecea et al., 1998; Sakurai et al., 1998) and have a critical role in arousal and sleep/ wakefulness (Chemelli et al., 1999; Lin et al., 1999; Tsujino and Sakurai, 2009; Berridge et al., 2010). $\mu$-Opioid receptors are expressed on lateral hypothalamic orexin neurons (Georgescu et al., 2003). Postmortem brains from persons diagnosed with heroin use disorder contained more orexin neurons than control brains (Thannickal et al., 2018). Orexin neurons originating in the lateral hypothalamus and projecting to the paraventricular nucleus of the thalamus (PVT) were able to mediate wakefulness. Chemogenetic inhibition of these orexin neurons decreased time spent awake and increased time spent in NREM in a 3-hour period (Ren et al., 2018). On the other hand, optogenetic stimulation of this pathway quickly induced wakefulness from NREM and REM (Ren et al., 2018).

In mice, 14 days (but not 7 days) of chronic morphine injections increased the number of immunohistochemically positive orexin cells throughout mouse brain (Thannickal et al., 2018). This 14-day morphine paradigm also increased relative mRNA expression of preprohypocretin and preprodynorphin. Preprohypocretin levels returned to baseline at 2 weeks of withdrawal from morphine (Thannickal et al., 2018). Long-term (60 days) injections at 10,25 , or $50 \mathrm{mg} / \mathrm{kg}$ morphine all increase the percentage of orexin cell number. The increase was greatest in the lateral hypothalamus, wherein orexin was synthesized, but morphine also increased

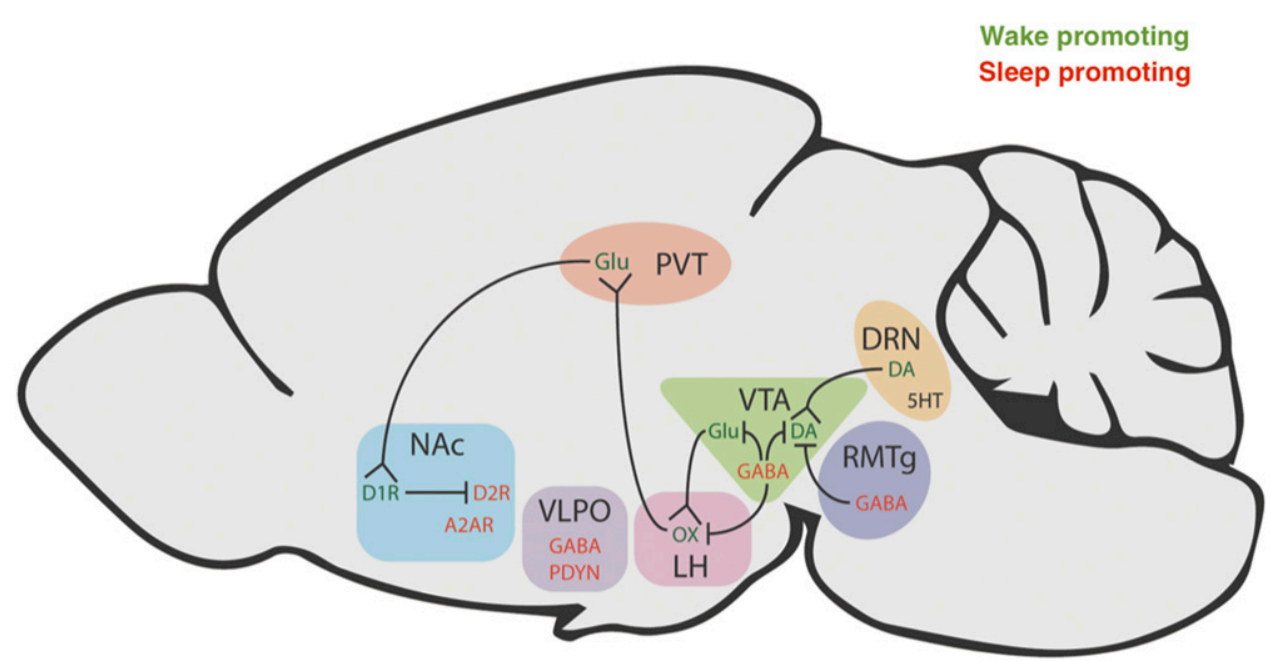

Fig. 2. Neural circuitry of reward/opioid regions involved in sleep/wake. A likely circuit mechanism regulating sleep/wake states that are engaged by opioid use. This figure infers the sleep/wake circuitry in response to opioids. Green indicates wake-promoting systems and red indicates sleep-promoting systems. Many brain regions were tested for neuronal activation or inhibition, but not all were tested for opioid effects on sleep. VLPO and RMTg neurons are sleep-promoting and are inhibited by opioids. This VLPO opioid exposure inhibits sleep-promoting neurons to produce wakefulness, whereas RMTg opioid exposure disinhibits VTA dopamine neurons. Connections between brain regions are drawn for studies in this review that have manipulated projections and examined sleep. For the VLPO, and for adenosine 2A receptor neurons in the nucleus accumbens, experiments examined manipulation of those neurons specifically, and projections to other reward-related brain regions were not established. 5HT, 5-hydroxytryptamine. DA, Dopamine. DRN, Dorsal Raphe Nucleus. 
orexin cell number in the medial hypothalamus (Thannickal et al., 2018). Fourteen days of chronic morphine injections kept orexin cell number elevated from control at 2 and 4 weeks of withdrawal, after which time orexin cell number returned to control levels (Thannickal et al., 2018). Interestingly, at 2 weeks after morphine withdrawal, despite increased orexin cell number, the size of each cell was smaller, and this effect went away by the 4 th week of withdrawal. In rats, a single $15 \mathrm{mg} / \mathrm{kg}$ injection of morphine increased both the action potential discharge in orexin neurons and activity and wakefulness measured by EEG and electromyography (Thannickal et al., 2018). Constitutive orexin knockout mice were narcoleptic (Chemelli et al., 1999). An inducible knockout mouse line was generated recently (Thannickal et al., 2018) to allow maintenance of narcolepsy but more cataplexy than constitutive orexin knockout mice. This more closely resembles narcolepsy in humans and allows for the treatment of cataplectic symptoms. These mice showed decreased orexin cell number compared with wild-type mice, but 14 days of morphine injections could reverse this and return orexin cell number to higher levels while also decreasing cataplexy (Thannickal et al., 2018). This study also showed that human patients with narcolepsy had very few orexin cells postmortem, but patients chronically treated with morphine for pain had increased orexin cell number compared with patients who were narcoleptic but still much less than control patients (Thannickal et al., 2018). Thus, data from immunohistochemistry, electrophysiology, and molecular analysis demonstrate an interaction of orexin and morphine resulting in increased wakefulness.

Orexin neurons increase their activity and peptide discharge in wakefulness compared with sleep (Thannickal et al., 2018) and express the immediate early gene c-Fos in response to morphine (Georgescu et al., 2003; Harris et al., 2007). Orexin neurons promote arousal when optogenetically activated (Adamantidis et al., 2007) and project to reward processing regions, such as the ventral tegmental area (Marcus et al., 2001). These projections to the VTA affect synaptic plasticity (Baimel and Borgland, 2015). Suvorexant is a dual orexin receptor antagonist the Food and Drug Administration-approved for the treatment of insomnia, but it is currently in a clinical trial for addiction (ClinicalTrials.gov identifier: NCT03412591). Thus, blocking orexin neurons with pharmacological therapeutics could be an avenue to treat morphine-induced sleep disruption.

Serotonin. The role of serotonin in sleep has been controversial, with early studies showing a sleep-promoting role (Mouret et al., 1968; Jouvet, 1969, 1972) and later experiments showing serotonin as a wake-activated neurotransmitter (Ursin, 2002; Lopez-Rodriguez et al., 2003; Zant et al., 2011). Serotonergic projections to the VTA excite VTA dopamine neurons (Wang et al., 2019), and rostral VTA neurons transmit GABA to disinhibit MOR-expressing serotonin cells in the dorsal raphe (Li et al., 2019). Studies examining direct serotonergic modulation of morphine-induced sleep/wake behaviors have not been done. However, a number of indirect studies show serotonin effects on sleep as well as serotonin effects on negative affect during withdrawal may contribute to opioid-mediated alterations in sleep/wake states.

Dorsal raphe serotonin neuron activity tracked with sleep/ wake states in mice as fiber photometry fluorescence showed increased activity in dorsal raphe serotonin neurons in wake states and decreased activity during sleep (Oikonomou et al., 2019). Lesioning dorsal raphe serotonergic cells in mice increased wakefulness and decreased NREM and REM, and optogenetic stimulation of these neurons increased wake probability with burst firing but decreased wake probability with tonic firing (Oikonomou et al., 2019). It is of interest that optogenetic activation of serotonin terminals projecting from the dorsal raphe to the VTA was rewarding. Activation of serotonergic dorsal raphe to VTA projections increased time spent in a CPP chamber paired with this stimulation (Wang et al., 2019). Chemogenetically activating dorsal raphe serotonin neurons shifts circadian timing of active/rest cycles and decreases probability of active states in the dark cycle while increasing the probability of active states in the light cycle (Urban et al., 2016).

Negative affect and sociability deficits are associated with protracted opiate withdrawal and are mediated by serotonin signaling. At 4 weeks of morphine withdrawal, serotonin turnover specifically in the dorsal raphe was increased, and the selective serotonin reuptake inhibitor fluoxetine reversed sociability deficits and immobility in the tail suspension test that resulted from morphine withdrawal (Goeldner et al., 2011). Tumor necrosis factor- $\alpha$ signaling from the lateral habenula projecting to the dorsal raphe was responsible for sociability deficits due to morphine withdrawal (Valentinova et al., 2019). Lastly, serotonin neurons can also project to orexin neurons and appear to regulate sleep architecture via 5-hydroxytryptamine 1a receptors (Saito et al., 2018). In this study, mice lacking 5-hydroxytryptamine 1a receptors exclusively in orexin neurons showed decreased wakefulness and increased NREM at the end of the dark cycle and also increased REM at the beginning of the light cycle (Saito et al., 2018) ). Further mechanistic studies are necessary to confirm the interaction of opioids in the serotonergic raphe system with sleep/wake and withdrawal behaviors.

Adenosine. Caffeine, the most widely used drug to increase alertness, acts by blocking adenosine receptors (Nehlig et al., 1992). Morphine, fentanyl, and buprenorphine decreased adenosine levels within the sleep-regulating pontine reticular formation and substantia innominata in rats (Nelson et al., 2009; Gauthier et al., 2011), whereas injecting adenosine into the pontine reticular formation promoted sleep in rats (Coleman et al., 2006). Adenosine also acted at adenosine $2 \mathrm{~A}$ receptors (A2ARs) in the VLPO to increase sleep (Scammell et al., 2001). Therefore, adenosine may be a critical regulator at the interface between disrupted sleep and opioid exposure [for review see Moore and Kelz (2009)]. Optogenetic and chemogenetic activation of A2AR-expressing neurons in the NAc core (but not shell) induces slow-wave sleep (Oishi et al., 2017). Inhibiting these NAc core A2AR neurons is wakepromoting and decreases slow-wave sleep. In addition, these neurons show less c-FOS expression in response to motivational stimuli (Oishi et al., 2017). Motivational stimuli, such as toys and chocolate, also decrease slow-wave sleep and c-FOS expression in these NAc A2AR neurons that project to the ventral pallidum (Oishi et al., 2017).

Glutamate. Glutamate neurons in the paraventricular thalamus mediate wakefulness. Multichannel electrophysiology showed that PVT glutamate neurons have a higher firing rate during wake compared with NREM and REM. Chemogenetic inhibition of these neurons decreases wakefulness and increases NREM and REM (Ren et al., 2018). 
Optogenetic stimulation of PVT glutamate neurons increases the probability of wake while decreasing the probability of NREM and decreases latency to wake both from sleeping and from isoflurane-induced anesthesia (Ren et al., 2018). PVT glutamate projections to the NAc are responsible for controlling wakefulness; however, those that project to the medial prefrontal cortex or insula do not have any impact on wake states (Ren et al., 2018). PVT neurons that project to the NAc also elicit c-FOS activation upon spontaneous withdrawal from morphine, and optogenetic inhibition of this pathway blocks somatic signs of opioid withdrawal (Zhu et al., 2016). Optogenetic stimulation of PVT glutamate terminals in the nucleus accumbens decreases latency to wake from both NREM and REM, and chemogenetic inhibition of this pathway decreases time spent in wake and increases time spent in NREM (Ren et al., 2018).

In addition to dopamine neurons, the VTA also contains GABA and glutamatergic neurons that were discovered via an unbiased screen to control arousal (Yu et al., 2019). These are mostly separate from VTA dopamine neurons-about $25 \%$ of glutamatergic neurons were $\mathrm{TH}+$, indicating only a small percentage of neurons have colocalized glutamate and dopamine, and the wakefulness phenotype driven by glutamate was maintained in the presence of dopamine antagonists (Yu et al., 2019). Glutamatergic neurons in the VTA promote wakefulness, whereas GABAergic neurons in the VTA promote sleep ( $\mathrm{Yu}$ et al., 2019). Selective chemogenetic or optogenetic stimulation of glutamatergic cells in the VTA produced wakefulness exclusively for a 5 -hour period. Calcium imaging showed that these neurons are more active during wake and REM than they are during NREM (Yu et al., 2019). Glutamate in these brain regions is a newly identified mechanism for regulating sleep/wake that is seen in opioidresponsive brain regions; however, direct effects of opioids on glutamate signaling in the VTA and PVT have not been tested.

GABA. GABAergic neurons in the VTA have a sleeppromoting role, as lesioning VTA GABA neurons elicits wakefulness (Yu et al., 2019). DREADDs stimulation of VTA GABA neurons decreases wake and increases NREM and REM, whereas DREADDs or optogenetic inhibition oppositely increase wake and decrease NREM and REM (Chowdhury et al., 2019; Yu et al., 2019). The mechanism proposed for VTA GABA neurons modulating wakefulness involves inhibiting nearby VTA dopamine and VTA glutamate neurons that both produce wakefulness (Yu et al., 2019). VTA GABA stimulation is behaviorally aversive and inhibits VTA dopamine (Yu et al., 2019). The RMTg is a sleep-promoting region that contains $\mu$-opioid receptors (Matsui et al., 2014). This nucleus receives input from the lateral habenula and is generally considered to be an inhibitory check on the midbrain dopamine system because RMTg GABA neurons project to and inhibit VTA dopamine neurons (Yang et al., 2018). Because VTA dopamine neurons promote wakefulness and RMTg GABA neurons inhibit VTA dopamine neurons, it follows that RMTg GABA neurons are sleep-promoting. Indeed, chemogenetic activation of RMTg neurons increases NREM sleep (Yang et al., 2018). This study used a virus to drive excitatory DREADDs expression in the RMTg, and although it was not targeted with a cell type-specific promoter, immunohistochemistry showed that nearly all (87\%) of the virally infected neurons were GABAergic (Yang et al., 2018). RMTg DREADDs stimulation increased NREM and decreased wakefulness and REM in 7 hours after clozapine $N$-oxide injection. Lesioning RMTg neurons with ibotenic acid increased wake and decreased NREM and REM in a 12-hour period at 8 and 16 days after the lesion (Yang et al., 2018). Optogenetic stimulation of RMTg terminals in the VTA was sufficient to inhibit VTA dopamine neurons from firing (Yang et al., 2018). Furthermore, DREADDs inhibition of VTA dopamine neurons decreases wakefulness and increases NREM without affecting REM sleep (Yang et al., 2018). Thus, the mechanism of opioids modulating wakefulness appears to be via sleeppromoting RMTg GABA neurons influencing midbrain dopamine neurons.

\section{Conclusion}

As described above, multiple groups of "reward" neurons impact sleep/wakefulness and are influenced by opioid receptor activation. Opioids induce both activation of wakepromoting systems and inhibition of sleep-promoting systems (Fig. 1). Additionally, wake-promoting and sleep-promoting circuits are mutually inhibitory. Thus, inhibiting or exciting any one of the regions will impact the others. Specifically, studies of optogenetic and chemogenetic manipulation of reward neurons that impact sleep/wakefulness provide a likely circuit that is engaged to promote wakefulness and reward upon opioid use by facilitating activation of wake-promoting systems and inhibition of sleep-promoting systems (Figs. 1 and 2). Based on these studies, a likely circuit involves opioid-mediated excitation of VTA dopamine neurons that are wake-promoting and projecting to the nucleus accumbens D1 receptor neurons. The sleep-promoting D2 receptor neurons and adenosine $2 \mathrm{~A}$ receptor neurons in the nucleus accumbens are inhibited. Lateral hypothalamus orexin neurons, which are wake-promoting, are excited by morphine and project to the paraventricular thalamus. The PVT may then send glutamatergic signals to the nucleus accumbens. Dorsal raphe dopamine neurons are influenced by opioids and project to VTA dopamine neurons. VTA GABA neurons as well as RMTg GABA neurons inhibit these midbrain dopamine neurons, whereas VTA glutamate neurons promote a role for wakefulness (Fig. 2). Clearly the effects of opioids on sleep are dose-, region-, and cell type-dependent, as high doses of opioids are sedative. Although some effort has been devoted to uncovering this, little is understood about the biology of opioid effects on sleep. The studies reviewed here have identified mechanisms associated with sleep, opioid response, and reward. More critical testing of key regions involved in opiate effects on sleep is much needed. For example, future studies could delete $\mu$-opioid receptors exclusively in the dopaminergic VTA or GABAergic VLPO neurons and examine whether the acute, chronic, and withdrawal effects of opioid administration are abolished. Optogenetic studies have been able to show immediate arousal from sleep states when reward-related neurons are activated, and chemogenetic studies have been able to extend arousal when animals would typically go back into a sleep state. However, to uncover the mechanism of chronic opioids on sleep and the effects of disrupted sleep on opioid use, optogenetics may be limiting by activation or inhibition on too short of a timescale. Future experiments could focus on chemogenetic manipulation of VTA neurons during chronic opioid use to evaluate the effects of these midbrain dopamine neurons on sleep and reward. The 
dorsal raphe may be implicated in opioid withdrawal and sleep, and chemogenetic manipulation in the dorsal raphe during withdrawal could be used to influence sleep and withdrawal-related behaviors. Finally, are opioid effects on sleep ever fully reversed, and if not, by what mechanisms is sleep persistently disrupted?

\section{Acknowledgments}

We thank Julia K. Brynildsen for artwork. The authors have no conflicts of interest to report. Authorship contributions: D.E. and J.A.B conceived of the topic and wrote the manuscript. S.C.V. contributed to writing and of the manuscript.

\section{References}

Adamantidis AR, Zhang F, Aravanis AM, Deisseroth K, and de Lecea L (2007) Neural substrates of awakening probed with optogenetic control of hypocretin neurons. Nature 450:420-424.

Alexandre C, Latremoliere A, Ferreira A, Miracca G, Yamamoto M, Scammell TE, and Woolf CJ (2017) Decreased alertness due to sleep loss increases pain sensitivity in mice. Nat Med 23:768-774.

Baimel C and Borgland SL (2015) Orexin signaling in the VTA gates morphineinduced synaptic plasticity. $J$ Neurosci 35:7295-7303 DOI: 10.1523/JNEUROSCI. 4385-14.2015.

Berridge CW, España RA, and Vittoz NM (2010) Hypocretin/orexin in arousal and stress. Brain Res 1314:91-102.

Beswick T, Best D, Rees S, Bearn J, Gossop M, and Strang J (2003) Major disruptions of sleep during treatment of the opiate withdrawal syndrome: differences between methadone and lofexidine detoxification treatments. Addict Biol 8:49-57.

Bonafide CP, Aucutt-Walter N, Divittore N, King T, Bixler EO, and Cronin AJ (2008) Remifentanil inhibits rapid eye movement sleep but not the nocturnal melatonin surge in humans. Anesthesiology 108:627-633.

Chemelli RM, Willie JT, Sinton CM, Elmquist JK, Scammell T, Lee C, Richardson JA, Williams SC, Xiong Y, Kisanuki Y, et al. (1999) Narcolepsy in orexin knockout mice: molecular genetics of sleep regulation. Cell 98:437-451.

Cho JR, Treweek JB, Robinson JE, Xiao C, Bremner LR, Greenbaum A, and Gradinaru V (2017) Dorsal raphe dopamine neurons modulate arousal and promote wakefulness by salient stimuli. Neuron 94:1205-1219.e8 DOI: 10.1016/j. neuron.2017.05.020.

Chowdhury S, Matsubara T, Miyazaki T, Ono D, Fukatsu N, Abe M, Sakimura K, Sudo Y, and Yamanaka A (2019) GABA neurons in the ventral tegmental area regulate non-rapid eye movement sleep in mice. eLife 8:e44928.

Chung S, Weber F, Zhong P, Tan CL, Nguyen TN, Beier KT, Hörmann N, Chang W-C, Zhang Z, Do JP, et al. (2017) Identification of preoptic sleep neurons using retrograde labelling and gene profiling. Nature 545:477-481.

Coffey AA, Guan Z, Grigson PS, and Fang J (2016) Reversal of the sleep-wake cycle by heroin self-administration in rats. Brain Res Bull 123:33-46.

Coleman CG, Baghdoyan HA, and Lydic R (2006) Dialysis delivery of an adenosine A2A agonist into the pontine reticular formation of C57BL/6J mouse increases pontine acetylcholine release and sleep. $J$ Neurochem 96:1750-1759.

Cronin A, Keifer JC, Baghdoyan HA, and Lydic R (1995) Opioid inhibition of rapid eye movement sleep by a specific mu receptor agonist. Br J Anaesth 74:188-192.

De Andrés I and Caballero A (1989) Chronic morphine administration in cats: effects on sleep and EEG. Pharmacol Biochem Behav 32:519-526.

de Lecea L, Kilduff TS, Peyron C, Gao X, Foye PE, Danielson PE, Fukuhara C, Battenberg EL, Gautvik VT, Bartlett FS II, et al. (1998) The hypocretins: hypothalamus-specific peptides with neuroexcitatory activity. Proc Natl Acad Sci USA 95:322-327.

Dimsdale JE, Norman D, DeJardin D, and Wallace MS (2007) The effect of opioids on sleep architecture. J Clin Sleep Med 3:33-36.

Durmer JS and Dinges DF (2005) Neurocognitive consequences of sleep deprivation. Semin Neurol 25:117-129.

Eban-Rothschild A, Rothschild G, Giardino WJ, Jones JR, and de Lecea L (2016) VTA dopaminergic neurons regulate ethologically relevant sleep-wake behaviors. Nat Neurosci 19:1356-1366.

Gauthier EA, Guzick SE, Brummett CM, Baghdoyan HA, and Lydic R (2011) Buprenorphine disrupts sleep and decreases adenosine concentrations in sleepregulating brain regions of Sprague Dawley rat. Anesthesiology 115:743-753.

Ge F, Mu P, Guo R, Cai L, Liu Z, Dong Y, and Huang YH (2019) Chronic sleep fragmentation enhances habenula cholinergic neural activity. Mol Psychiatry DOI: 10.1038/s41380-019-0419-z [published ahead of print]

Georgescu D, Zachariou V, Barrot M, Mieda M, Willie JT, Eisch AJ, Yanagisawa M, Nestler EJ, and Dileone RJ (2003) Involvement of the lateral hypothalamic peptide orexin in morphine dependence and withdrawal. $J$ Neurosci 23:3106-3111.

Glick SD, Ramirez RL, Livi JM, and Maisonneuve IM (2006) 18-Methoxycoronaridine acts in the medial habenula and/or interpeduncular nucleus to decrease morphine self-administration in rats. Eur $J$ Pharmacol 537:94-98 DOI: 10.1016/j.ejphar. 2006.03.045

Goeldner C, Lutz P-E, Darcq E, Halter T, Clesse D, Ouagazzal A-M, and Kieffer BL (2011) Impaired emotional-like behavior and serotonergic function during protracted abstinence from chronic morphine. Biol Psychiatry 69:236-244.

Greco MA, Fuller PM, Jhou TC, Martin-Schild S, Zadina JE, Hu Z, Shiromani P, and $\mathrm{Lu} \mathrm{J}$ (2008) Opioidergic projections to sleep-active neurons in the ventrolateral preoptic nucleus. Brain Res 1245:96-107.
Harris GC, Wimmer M, Randall-Thompson JF, and Aston-Jones G (2007) Lateral hypothalamic orexin neurons are critically involved in learning to associate an environment with morphine reward. Behav Brain Res 183:43-51.

Hartwell EE, Pfeifer JG, McCauley JL, Moran-Santa Maria M, and Back SE (2014) Sleep disturbances and pain among individuals with prescription opioid dependence. Addict Behav 39:1537-1542.

Hedegaard H, Miniño AM, and Warner M; National Center for Health Statistics (2020) Drug overdose deaths in the United States, 1999-2018 no. 356, NCHS Data Brief, Hyattsville, MD

Howe RC, Phillips JL, and Hegge FW (1981) Acute heroin abstinence in man: IV Sleep--waking state contingencies. Drug Alcohol Depend 7:163-176.

Jouvet M (1969) Biogenic amines and the states of sleep. Science 163:32-41.

Jouvet M (1972) The role of monoamines and acetylcholine-containing neurons in the regulation of the sleep-waking cycle. Ergeb Physiol 64:166-307.

Keifer JC, Baghdoyan HA, and Lydic R (1992) Sleep disruption and increased apneas after pontine microinjection of morphine. Anesthesiology 77:973-982.

Khazan N and Colasanti B (1972) Protracted rebound in rapid movement sleep time and electroencephalogram voltage output in morphine-dependent rats upon withdrawal. J Pharmacol Exp Ther 183:23-30.

Lewis SA, Oswald I, Evans JI, Akindele MO, and Tompsett SL (1970) Heroin and human sleep. Electroencephalogr Clin Neurophysiol 28:374-381.

Li C, Sugam JA, Lowery-Gionta EG, McElligott ZA, McCall NM, Lopez AJ, McKlveen JM, Pleil KE, and Kash TL (2016) Mu opioid receptor modulation of dopamine neurons in the periaqueductal gray/dorsal raphe: a role in regulation of pain Neuropsychopharmacology 41:2122-2132.

Li Y, Li C-Y, Xi W, Jin S, Wu Z-H, Jiang P, Dong P, He X-B, Xu F-Q, Duan S, et al. (2019) Rostral and caudal ventral tegmental area GABAergic inputs to different dorsal raphe neurons participate in opioid dependence. Neuron 101:748-761.e5.

Lim J and Dinges DF (2008) Sleep deprivation and vigilant attention. Ann N Y Acad Sci 1129: 305-322.

Lin L, Faraco J, Li R, Kadotani H, Rogers W, Lin X, Qiu X, de Jong PJ, Nishino S, and Mignot $\mathrm{E}$ (1999) The sleep disorder canine narcolepsy is caused by a mutation in the hypocretin (orexin) receptor 2 gene. Cell 98:365-376.

Logan RW, Hasler BP, Forbes EE, Franzen PL, Torregrossa MM, Huang YH, Buysse DJ, Clark DB, and McClung CA (2018) Impact of sleep and circadian rhythms on addiction vulnerability in adolescents. Biol Psychiatry 83:987-996.

Lopez-Rodriguez F, Wilson CL, Maidment NT, Poland RE, and Engel J (2003) Total sleep deprivation increases extracellular serotonin in the rat hippocampus. Neuroscience 121:523-530.

Louter M, Aarden WCCA, Lion J, Bloem BR, and Overeem S (2012) Recognition and diagnosis of sleep disorders in Parkinson's disease. J Neurol 259:2031-2040.

Luo YJ, Li YD, Wang L, Yang SR, Yuan XS, Wang J, Cherasse Y, Lazarus M, Chen JF, Qu WM, et al. (2018) Nucleus accumbens controls wakefulness by a subpopulation of neurons expressing dopamine $\mathrm{D}_{1}$ receptors. Nat Commun 9:1576.

Lydic R, Keifer JC, Baghdoyan HA, and Becker L (1993) Microdialysis of the pontine reticular formation reveals inhibition of acetylcholine release by morphine. Anesthesiology 79:1003-1012.

Lydon-Staley DM, Cleveland HH, Huhn AS, Cleveland MJ, Harris J, Stankoski D, Deneke E, Meyer RE, and Bunce SC (2017) Daily sleep quality affects drug craving, partially through indirect associations with positive affect, in patients in treatment for nonmedical use of prescription drugs. Addict Behav 65:275-282.

Marcus JN, Aschkenasi CJ, Lee CE, Chemelli RM, Saper CB, Yanagisawa M, and Elmquist JK (2001) Differential expression of orexin receptors 1 and 2 in the rat brain. $J$ Comp Neurol 435:6-25.

Margarit C, Ballester P, Inda MD, Roca R, Gomez L, Planelles B, Ajo R, Morales D, and Peiró AM (2019) OPRM1 gene interaction with sleep in chronic pain patients treated with opioids. Pain Physician 22:97-107.

Martin WR, Jasinski DR, Haertzen CA, Kay DC, Jones BE, Mansky PA and Carpenter RW (1973) Methadone--a reevaluation. Arch Gen Psychiatry 28: 286-295.

Matsui A, Jarvie BC, Robinson BG, Hentges ST, and Williams JT (2014) Separate GABA afferents to dopamine neurons mediate acute action of opioids, development of tolerance, and expression of withdrawal. Neuron 82:1346-1356.

Maulik PK, Tripathi BM, and Pal HR (2002) Coping behaviors and relapse precipitants in opioid dependence: a study from North India. J Subst Abuse Treat 22 135-140.

Mazei-Robison MS, Koo JW, Friedman AK, Lansink CS, Robison AJ, Vinish M Krishnan V, Kim S, Siuta MA, Galli A, et al. (2011) Role for mTOR signaling and neuronal activity in morphine-induced adaptations in ventral tegmental area dopamine neurons. Neuron 72:977-990 DOI: 10.1016/j.neuron.2011.10.012.

McDevitt RA, Tiran-Cappello A, Shen H, Balderas I, Britt JP, Marino RAM, Chung SL, Richie CT, Harvey BK, and Bonci A (2014) Serotonergic versus nonserotonergic dorsal raphe projection neurons: differential participation in reward circuitry. Cell Rep 8:1857-1869.

Moore JT and Kelz MB (2009) Opiates, sleep, and pain: the adenosinergic link. Anesthesiology 111:1175-1176.

Mouret J, Bobillier P, and Jouvet M (1968) Insomnia following parachlorophenylalanine in the rat. Eur J Pharmacol 5:17-22

Nascimento DC, Andersen ML, Hipólide DC, Nobrega JN, and Tufik S (2007) Pain hypersensitivity induced by paradoxical sleep deprivation is not due to altered binding to brain $\mu$-opioid receptors. Behav Brain Res 178:216-220.

Nehlig A, Daval JL, and Debry G (1992) Caffeine and the central nervous system: mechanisms of action, biochemical, metabolic and psychostimulant effects. Brain Res Brain Res Rev 17:139-170.

Nelson AM, Battersby AS, Baghdoyan HA, and Lydic R (2009) Opioid-induced decreases in rat brain adenosine levels are reversed by inhibiting adenosine deaminase. Anesthesiology 111:1327-1333.

Nestler EJ (2004) Molecular mechanisms of drug addiction. Neuropharmacology 47 (Suppl 1):24-32. 
Oikonomou G, Altermatt M, Zhang R-W, Coughlin GM, Montz C, Gradinaru V, and Prober DA (2019) The serotonergic raphe promote sleep in Zebrafish and mice. Neuron 103:686-701.e8.

Oishi Y, Xu Q, Wang L, Zhang BJ, Takahashi K, Takata Y, Luo YJ, Cherasse Y, Schiffmann SN, de Kerchove d'Exaerde A, et al. (2017) Slow-wave sleep is controlled by a subset of nucleus accumbens core neurons in mice. Nat Commun 8:734.

Onen SH, Alloui A, Gross A, Eschallier A, and Dubray C (2001) The effects of total sleep deprivation, selective sleep interruption and sleep recovery on pain tolerance thresholds in healthy subjects. J Sleep Res 10:35-42.

Ren S, Wang Y, Yue F, Cheng X, Dang R, Qiao Q, Sun X, Li X, Jiang Q, Yao J, et al. (2018) The paraventricular thalamus is a critical thalamic area for wakefulness. Science 362:429-434

Roehrs T, Hyde M, Blaisdell B, Greenwald M, and Roth T (2006) Sleep loss and REM sleep loss are hyperalgesic. Sleep 29:145-151.

Roehrs T and Roth T (2005) Sleep and pain: interaction of two vital functions. Semin Neurol 25:106-116.

Sagaspe P, Sanchez-Ortuno M, Charles A, Taillard J, Valtat C, Bioulac B, and Philip P (2006) Effects of sleep deprivation on Color-Word, Emotional, and Specific Stroop interference and on self-reported anxiety. Brain Cogn 60:76-87.

Saito YC, Tsujino N, Abe M, Yamazaki M, Sakimura K, and Sakurai T (2018) Serotonergic input to orexin neurons plays a role in maintaining wakefulness and REM sleep architecture. Front Neurosci 12:892.

Sakurai T, Amemiya A, Ishii M, Matsuzaki I, Chemelli RM, Tanaka H, Williams SC, Richardson JA, Kozlowski GP, Wilson S, et al. (1998) Orexins and orexin receptors: a family of hypothalamic neuropeptides and $G$ protein-coupled receptors that regulate feeding behavior. Cell 92:573-585.

Scammell TE, Gerashchenko DY, Mochizuki T, McCarthy MT, Estabrooke IV, Sears CA, Saper CB, Urade Y, and Hayaishi O (2001) An adenosine A2a agonist increases sleep and induces Fos in ventrolateral preoptic neurons. Neuroscience 107:653-663.

Scholl L, Seth P, Kariisa M, Wilson N, and Baldwin G (2018) Drug and opioidinvolved overdose deaths - United States, 2013-2017. MMWR Morb Mortal Wkly Rep 67:1419-1427.

Sharkey KM, Kurth ME, Corso RP, Brower KJ, Millman RP, and Stein MD (2009) Home polysomnography in methadone maintenance patients with subjective sleep complaints. Am J Drug Alcohol Abuse 35:178-182.

Shaw IR, Lavigne G, Mayer P, and Choinière M (2005) Acute intravenous administration of morphine perturbs sleep architecture in healthy pain-free young adults: a preliminary study [published correction appears in Sleep (2006) 29:136]. Sleep 28:677-682.

Skinner GO, Damasceno F, Gomes A, and de Almeida OMMS (2011) Increased pain perception and attenuated opioid antinociception in paradoxical sleep-deprived rats are associated with reduced tyrosine hydroxylase staining in the periaqueductal gray matter and are reversed by L-dopa. Pharmacol Biochem Behav 99:94-99.

Stein MD, Herman DS, Bishop S, Lassor JA, Weinstock M, Anthony J, and Anderson BJ (2004) Sleep disturbances among methadone maintained patients. J Subst Abuse Treat 26:175-180.

Taylor NE, Van Dort CJ, Kenny JD, Pei J, Guidera JA, Vlasov KY, Lee JT, Boyden ES, Brown EN, and Solt K (2016) Optogenetic activation of dopamine neurons in the ventral tegmental area induces reanimation from general anesthesia. Proc Natl Acad Sci USA 113:12826-12831.

Thannickal TC, John J, Shan L, Swaab DF, Wu MF, Ramanathan L, McGregor R, Chew KT, Cornford M, Yamanaka A, et al. (2018) Opiates increase the number of hypocretin-producing cells in human and mouse brain and reverse cataplexy in a mouse model of narcolepsy. Sci Transl Med 10:1-14.
Tomim DH, Pontarolla FM, Bertolini JF, Arase M, Tobaldini G, Lima MMS, and Fischer L (2016) The pronociceptive effect of paradoxical sleep deprivation in rats: evidence for a role of descending pain modulation mechanisms. Mol Neurobiol 53:1706-1717.

Tsujino N and Sakurai T (2009) Orexin/hypocretin: a neuropeptide at the interface of sleep, energy homeostasis, and reward system. Pharmacol Rev 61:162-176 DOI: 10.1124/pr.109.001321.

Urban DJ, Zhu H, Marcinkiewcz CA, Michaelides M, Oshibuchi H, Rhea D, Aryal DK, Farrell MS, Lowery-Gionta E, Olsen RH, et al. (2016) Elucidation of the behavioral program and neuronal network encoded by dorsal raphe serotonergic neurons. Neuropsychopharmacology 41:1404-1415.

Ursin R (2002) Serotonin and sleep. Sleep Med Rev 6:55-69.

Valentinova K, Tchenio A, Trusel M, Clerke JA, Lalive AL, Tzanoulinou S, Matera A, Moutkine I, Maroteaux L, Paolicelli RC, et al. (2019) Morphine withdrawal recruits lateral habenula cytokine signaling to reduce synaptic excitation and sociability. Nat Neurosci 22:1053-1056 DOI: 10.1038/s41593-019-0421-4.

Vowles KE, McEntee ML, Julnes PS, Frohe T, Ney JP, and Van Der Goes DN (2015) Rates of opioid misuse, abuse, and addiction in chronic pain: a systematic review and data synthesis. Pain 156:596-576.

Wang HL, Zhang S, Qi J, Wang H, Cachope R, Mejias-Aponte CA, Gomez JA, MateoSemidey GE, Beaudoin GMJ, Paladini CA, et al. (2019) Dorsal raphe dual serotonin-glutamate neurons drive reward by establishing excitatory synapses on VTA mesoaccumbens dopamine neurons. Cell Rep 26:1128-1142.e7.

Wang Q, Yue XF, Qu WM, Tan R, Zheng P, Urade Y, and Huang ZL (2013) Morphine inhibits sleep-promoting neurons in the ventrolateral preoptic area via mu receptors and induces wakefulness in rats. Neuropsychopharmacology 38: 791-801.

Weaver MD, Barger LK, Malone SK, Anderson LS, and Klerman EB (2018) Dosedependent associations between sleep duration and unsafe behaviors among US high school students. JAMA Pediatr 172:1187-1189.

Wells AM, Ridener E, Bourbonais CA, Kim W, Pantazopoulos H, Carroll FI, Kim K-S, Cohen BM, and Carlezon WA Jr (2017) Effects of chronic social defeat stress on sleep and circadian rhythms are mitigated by kappa-opioid receptor antagonism. $J$ Neurosci 37:7656-7668.

Xiao L, Tang YL, Smith AK, Xiang YT, Sheng LX, Chi Y, Du WJ, Guo S, Jiang ZN, Zhang GF, et al. (2010) Nocturnal sleep architecture disturbances in early methadone treatment patients. Psychiatry Res 179:91-95.

Yang SR, Hu ZZ, Luo YJ, Zhao YN, Sun HX, Yin D, Wang CY, Yan YD, Wang DR, Yuan XS, et al. (2018) The rostromedial tegmental nucleus is essential for nonrapid eye movement sleep. PLoS Biol 16:e2002909.

Yu X, Li W, Ma Y, Tossell K, Harris JJ, Harding EC, Ba W, Miracca G, Wang D, Li L, et al. (2019) GABA and glutamate neurons in the VTA regulate sleep and wakefulness. Nat Neurosci 22:106-119.

Zant JC, Leenaars CHC, Kostin A, Van Someren EJ, and Porkka-Heiskanen T (2011) Increases in extracellular serotonin and dopamine metabolite levels in the basal forebrain during sleep deprivation. Brain Res 1399:40-48.

Zhu Y, Wienecke CFR, Nachtrab G, and Chen X (2016) A thalamic input to the nucleus accumbens mediates opiate dependence. Nature 530:219-222.

Address correspondence to: Julie A. Blendy, Department of Systems Pharmacology and Translational Therapeutics, Perelman School of Medicine, University of Pennsylvania, Translational Research Laboratory, 125 S. 31st St., Philadelphia, PA 19104. E-mail: blendy@pennmedicine.upenn.edu 